\section{Rare and Fascinating Case of ST-Elevation Myocardial Infarction Diagnosis From an Underlying Ventricular Paced Rhythm}

\author{
Woosun Kang ${ }^{1}$, Liang D. Ge ${ }^{1}$, Puja Patel ${ }^{2}$, Raj Patel ${ }^{3}$, Tinoy Kizhakekuttu ${ }^{3}$ \\ 1. Internal Medicine, University of Illinois College of Medicine at Peoria, Peoria, USA 2. Internal Medicine, American \\ University of Antigua, Peoria, USA 3. Cardiology, University of Illinois College of Medicine at Peoria, Peoria, USA
}

Corresponding author: Raj Patel, rdpatel@carilionclinic.org

\section{Abstract}

This is a case of a patient diagnosed with anterior ST-elevation myocardial infarction (STEMI) with a ventricular paced rhythm after the patient underwent a femoral-femoral bypass surgery for severe peripheral vascular disease. The case highlights the diagnosis of STEMI in the setting of paced rhythm in the appropriate clinical setting.

Categories: Cardiology, Internal Medicine, Medical Education

Keywords: ventricular paced rhythm, electrophysiology, cardiac catheterization, acute coronary syndrome, cardiology, cardiology research

\section{Introduction}

It is difficult to diagnose ST-elevation myocardial infarction (STEMI) in the setting of an abnormal baseline EKG, such as a left bundle branch block (LBBB). Fortunately, the Sgarbossa criteria have been accepted as a highly specific (96\%) algorithm for the diagnosis of STEMI with concurrent LBBB [1]. Patients who are paced in the right ventricle manifest iatrogenic LBBB. Thus, the Sgarbossa criteria can be used as a tool for STEMI diagnosis in the setting of right ventricular paced rhythm (VPR) [2-4]. This case highlights the clinical decision-making for the diagnosis of STEMI in a patient with VPR after recent femoral-femoral bypass surgery for severe peripheral vascular disease.

\section{Case Presentation}

This is the case of an 83-year-old male with a medical history of essential hypertension, hyperlipidemia, ischemic cardiomyopathy, coronary artery disease (CAD), PVD, and abdominal aortic aneurysm. The patient has a known history of bi-ventricular implantable cardioverter defibrillator implantation and aortic aneurysm repair. She presented to the emergency department with typical angina. Two weeks prior to presentation, the patient was discharged after femoral-femoral bypass surgery for severe lower extremity PVD. Presenting EKG revealed concordant > $1 \mathrm{~mm}$ ST-segment elevations in the anterior and inferior leads (Figure 1), which were new compared to baseline EKG (Figure 2). Initial vitals were as follows: temperature of $98.2^{\circ} \mathrm{F}$, pulse rate of 74 , blood pressure of $130 / 76$, respiratory rate of 20 breaths per minute, and $\mathrm{SpO}_{2}$ of $97 \%$ on room air. Physical examination revealed bilateral lung rales and 1+ bilateral lower extremity edema. No jugular venous distension or carotid bruits were noted and peripheral pulses were symmetric, 2+, and intact. Initial troponin was elevated at 0.111 . Urgent transthoracic echocardiogram (TTE) showed visually decreased left ventricular ejection fraction (LVEF) of 30-35\%, which was reduced from a baseline of 40-45\%. Additionally, basal-to-inferior and mid-inferior wall akinesis was noted, as well as severely hypokinetic midto-apical anterior and anteroseptal walls. 


\section{Cureus}

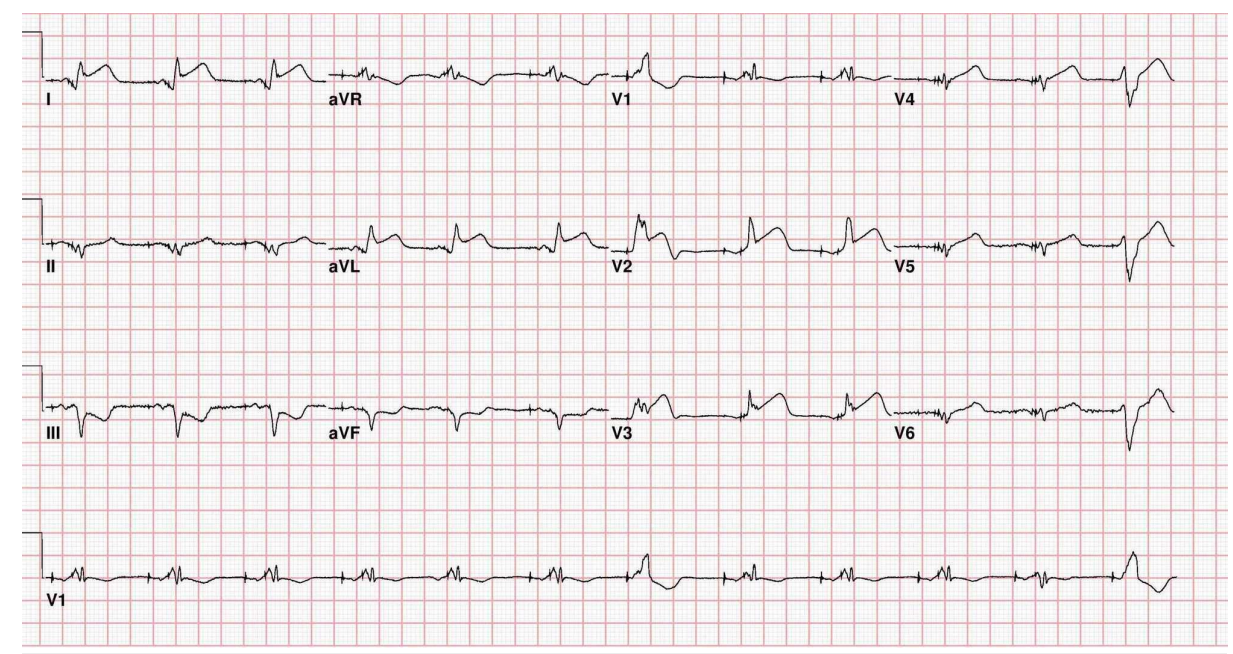

FIGURE 1: Presenting EKG revealing ventricular paced rhythm with ST elevations in the anterior and lateral leads with inferior depression.

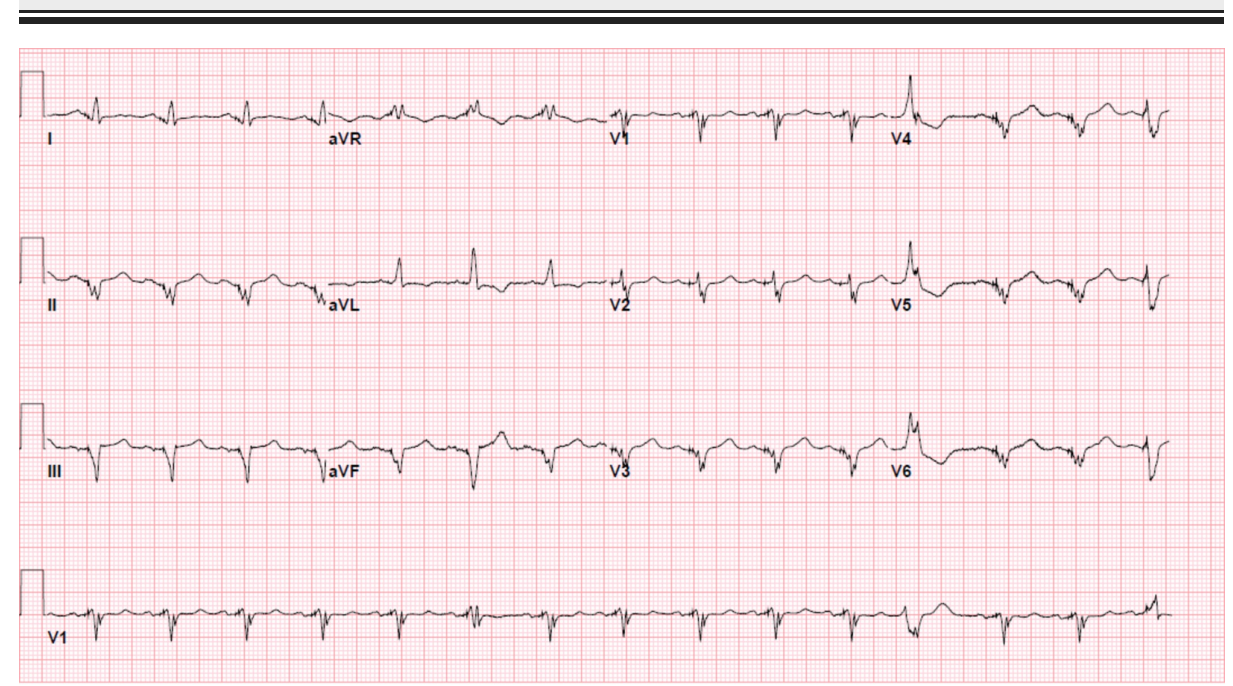

FIGURE 2: Baseline EKG several months prior to presentation for STEMI.

STEMI, ST-elevation myocardial infarction

HHeparin drip was initiated in the emergency room, and the patient was taken for emergent cardiac catheterization/coronary angiography. A loading dose of ticagrelor and aspirin was administered prior to angiography. The patient was noted to have $90 \%$ ulcerated plaque of the proximal left anterior descending artery (Figure 3), which was successfully treated with a $3.0 \times 18 \mathrm{~mm}$ XIENCE Sierra drug-eluting stent (Abbott, Abbott Park, IL, USA), reducing the 90\% lesion to 0\%, with post-PCI TIMI III flow noted (Figure 4). His anginal symptoms resolved after percutaneous coronary intervention (PCI). EKG abnormalities resolved with troponin peaking at 90.431 after the PCI. Medical therapy was optimized for CAD and acute systolic dysfunction prior to discharge from the hospital. 


\section{Cureus}

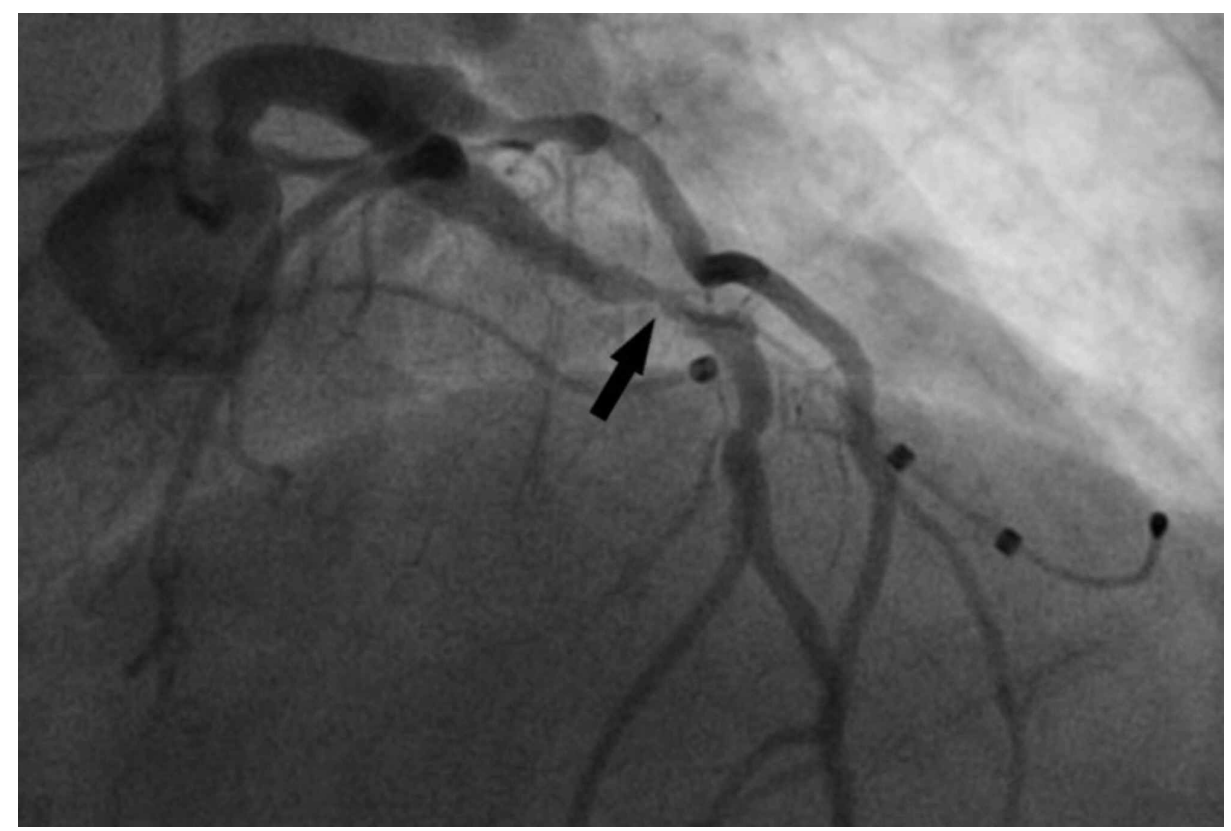

FIGURE 3: Severe proximal left anterior descending artery stenosis with an acute, ulcerated appearance.

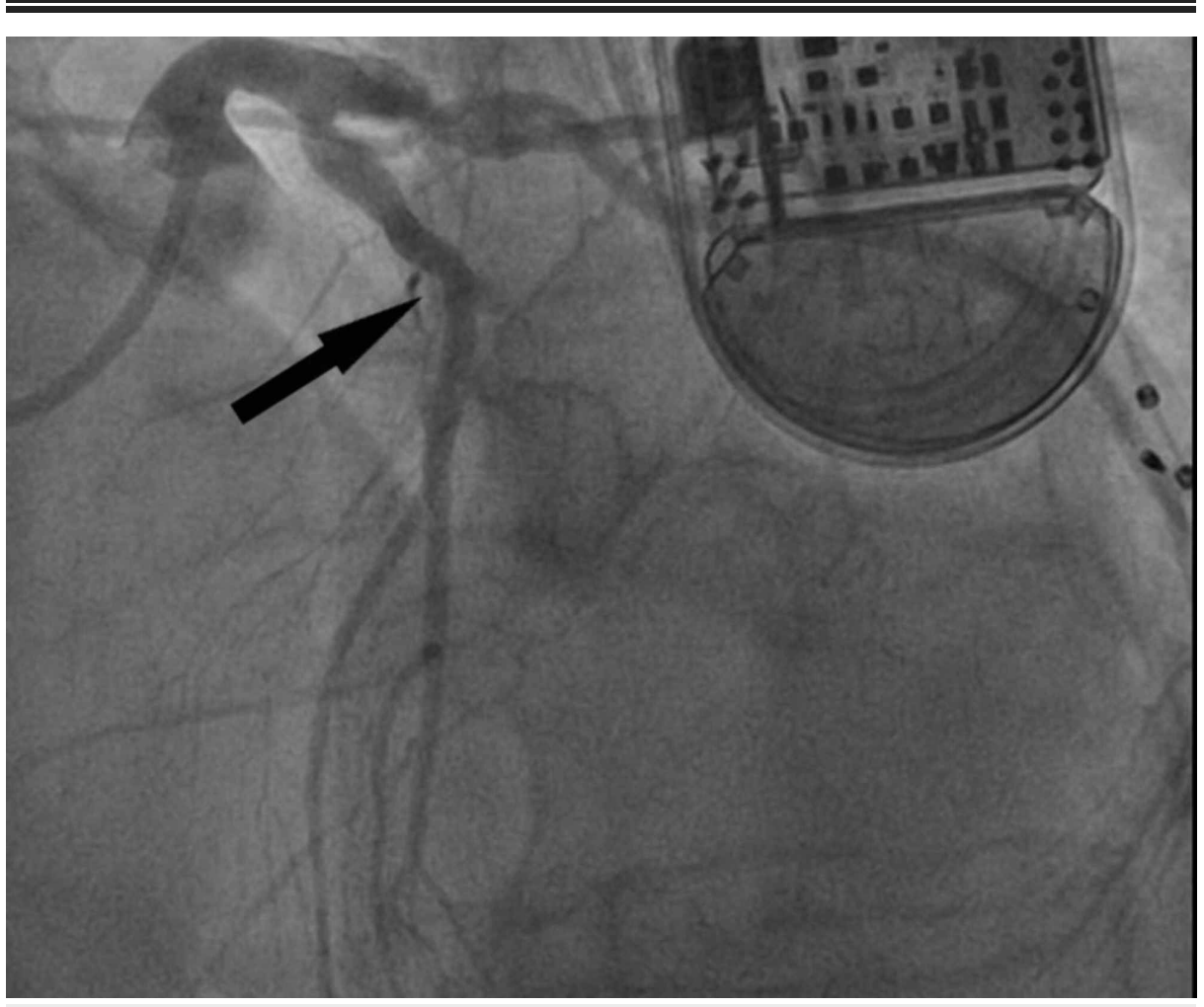

FIGURE 4: Arrow showing a successful PCI with a drug-eluting stent to the proximal left anterior descending artery.

$\mathrm{PCl}$, percutaneous coronary intervention

\section{Discussion}

In the subset of patients with VPR, diagnosis of STEMI can be challenging. Currently, there are no validated diagnostic criteria recognized for the diagnosis of STEMI on VPR [1,2]. It has been suggested that having diagnostic criteria for STEMI in patients with VPR will lead to early reperfusion and better clinical 
outcomes $[2,4,5]$. LBBB is not a rare finding in patients with myocardial infarction (MI); however, the proportion of patients with LBBB and chest pain who actually have MI varies between $13 \%$ and $32 \%$ [1,6-8]. In addition, almost one-half of patients with LBBB and MI do not have chest pain, which further emphasizes the importance of an accurate diagnostic tool [9].

Previously described EKG criteria may be used to predict STEMI in patients with VPR and LBBB. The Sgarbossa criteria from the GUSTO-1 (Global Utilization of t-PA and Streptokinase for Occluded Coronary Arteries) trial have been shown to be highly specific for acute STEMI in the setting of known LBBB1 (Figure 5). A higher Sgarbossa score is correlated to increased mortality after STEMI and increased severity of MI [10]. The dilemma is that only $0.1 \%$ patients in GUSTO- 1 trial had VPR, meaning there are insufficient data to extrapolate the results to VPR patients [1]. The Sgarbossa criteria can be used alongside the Smithmodified Sgarbossa criteria, which takes into account discordant STE with an amplitude of $\geqslant 25 \%$ of the depth of the preceding S-wave [1,11]. It remains controversial how effective these criteria are in the setting of VPR. Future studies should be directed at patients meeting the STEMI criteria on VPRs and the use of/utility of the aforementioned criteria in the diagnosis of acute STEMI.

\section{The Sgarbossa Criteria}

1. Concordant $\mathrm{ST}$ elevation $\geq 1 \mathrm{~mm}$ in a lead with a positive QRS complex (5 points)

2. ST depression $\geq 1 \mathrm{~mm}$ in V1, V2, or V3 (3 points)

3. Discordant $\mathrm{ST}$ elevation $\geq 5 \mathrm{~mm}$ in a lead with a negative QRS complex (2 points)

Scoring: $\geq 3$ points is highly specific (96\%) for acute coronary syndrome in patients with LBBB $^{1}$

\section{FIGURE 5: Commonly used Scarbossa criteria for the diagnosis of STEMI in the setting of LBBB.}

STEMI, ST-elevation myocardial infarction; LBBB, left bundle branch block

\section{Conclusions}

This case study illustrates how the Sgarbossa criteria along with good clinical acumen can potentially be applied to VPR and lead to the diagnosis of STEMI. In addition to EKG changes, the patient's presenting symptoms, laboratory abnormalities, and echocardiogram were all taken into account to reach the diagnosis of STEMI.

\section{Additional Information \\ Disclosures}

Human subjects: Consent was obtained by all participants in this study. Conflicts of interest: In compliance with the ICMJE uniform disclosure form, all authors declare the following: Payment/services info: All authors have declared that no financial support was received from any organization for the submitted work. Financial relationships: All authors have declared that they have no financial relationships at present or within the previous three years with any organizations that might have an interest in the submitted work. Other relationships: All authors have declared that there are no other relationships or activities that could appear to have influenced the submitted work.

\section{References}

1. Sgarbossa EB, Pinski SL, Barbagelata A: Electrocardiographic diagnosis of evolving acute myocardial infarction in the presence of left bundle branch block. GUSTO-1 (Global Utilization of Streptokinase and Tissue Plasminogen Activator for Occluded Coronary Arteries) Investigators. N Engl J Med. 1996, 334:481487. 10.1056/NEJM199602223340801

2. Bertel N, Witassek F, Puhan M, et al.: Management and outcomes of patients with acute myocardial infarction presenting with pacemaker rhythm. Int J Cardiol. 2017, 230:604-609. 10.1016/j.ijcard.2016.12.047

3. Karumbaiah K, Omar B: ST-elevation myocardial infarction in the presence of biventricular paced rhythm. J Emerg Med. 2013, 45:35-40. 10.1016/j.jemermed.2013.03.034 
4. Jackson M, Mahmood NM: Chest pain in a patient with left bundle branch block . JAMA Intern Med. 2016, 176:1211-1212. 10.1001/jamainternmed.2016.3225

5. Brodie BR, Stone GW, Cox DA, et al.: Impact of treatment delays on outcomes of primary percutaneous coronary intervention for acute myocardial infarction: analysis from the CADILLAC trial. Am Heart J. 2006, 151:1231-1238. 10.1016/j.ahj.2005.07.016

6. Go AS, Barron HV, Rundle AC, Ornato JP, Avins AL: Bundle-branch block and in-hospital mortality in acute myocardial infarction. National Registry of Myocardial Infarction 2 Investigators. Ann Intern Med. 1998, 129:690-697. 10.7326/0003-4819-129-9-199811010-00003

7. Kontos MC, McQueen RH, Jesse RL, Tantum JL, Ornato JP: Can myocardial infarction be rapidly identified in emergency department patients who have left bundle-branch block?. Ann Emerg Med. 2001, 37:431-438. 10.1067/mem.2001.114900

8. Shlipak MG, Lyons WL, Go AS, Chou TM, Evans Gt, Browner WS: Should the electrocardiogram be used to guide therapy for patients with left bundle-branch block and suspected myocardial infarction?. JAMA. 1999, 281:714-719. 10.1001/jama.281.8.714

9. Shlipak MG, Go AS, Frederick PD, Malmgren J, Barron HV, Canto JG: Treatment and outcomes of left bundle-branch block patients with myocardial infraction who present without chest pain. National Registry of Myocardial Infarction 2 Investigators. J Am Coll Cardiol. 2000, 36:706-712. 10.1016/s07351097(00)00789-0

10. Al-Faleh H, Fu Y, Wagner G, et al.: Unraveling the spectrum of left bundle branch block in acute myocardial infarction: Insights from the Assessment of the Safety and Efficacy of a New Thrombolytic (ASSENT 2 and 3) trials. 2006, 3:10-15. 10.1016/j.ahj.2005.02.043

11. Smith SW, Dodd KW, Henry TD, Dvorak DM, Pearce LA: Diagnosis of ST elevation myocardial infarction in the presence of left bundle branch block using the ST elevation to S-wave ratio in a modified Sgarbossa rule. Ann Emerg Med. 2012, 60:766-776. 10.1016/j.annemergmed.2012.07.119 